\section{Caelyx beim metastasierten Mamma- karzinom zugelassen: Neue effektive Therapieoption mit besserer Verträglichkeit dank neuer Galenik}

Etwa jede 8. bis 10. Frau in der westlichen Welt erkrankt an einem Mammakarzinom. Bei rund $60 \%$ aller neu diagnostizierten Brustkrebsfälle treten im Verlauf der Erkrankung Metastasen auf. «Auf Grund der heute verfügbaren Medikamente beobachten wir bei einer Vielzahl der Frauen mit Mammakarzinom einen oft jahrelangen chronifizierten Krankheitsverlauf. Unter diesem Aspekt ist es besonders wichtig, dass uns Medikamente zur Verfügung stehen, die die Lebensqualität der Patientinnen so gut wie möglich aufrecht erhalten.» Mit diesen Worten verwies Professor Siegfried Seeber vom Universitätsklinikum Essen auf den hohen Nutzen der pegylierten liposomalen Formulierung von Doxorubicin (Caelyx) anlässlich der EinführungsPressekonferenz der Essex Pharma Ende Januar 2003 in München. Denn diese innovative Formulierung bietet gegenüber dem konventio- nellen Doxorubicin den Vorteil, wesentlich nebenwirkungsärmer zu sein. Sie dient damit dem Ziel einer guten Lebensqualität.

\section{Entscheidende Unterschiede gegen- über konventionellen Anthrazyklinen}

«Obwohl Doxorubicin beim metastasierten Mammakarzinom unter die wirksamsten Zytostatika einzustufen ist, wirkt die starke Toxizität doch immer einsatzbegrenzend», berichtete Professor Carsten Bokemeyer, Universitätsklinikum Tübingen. Neben akuten Nebenwirkungen wie Mukositis, Alopezie sowie Übelkeit und Erbrechen kommt es zu schweren nicht-reversiblen Herzschädigungen. Dies gilt besonders bei Patientinnen, bei denen bereits ein kardiales Risiko vorliegt. Bokemeyer verwies darauf, dass

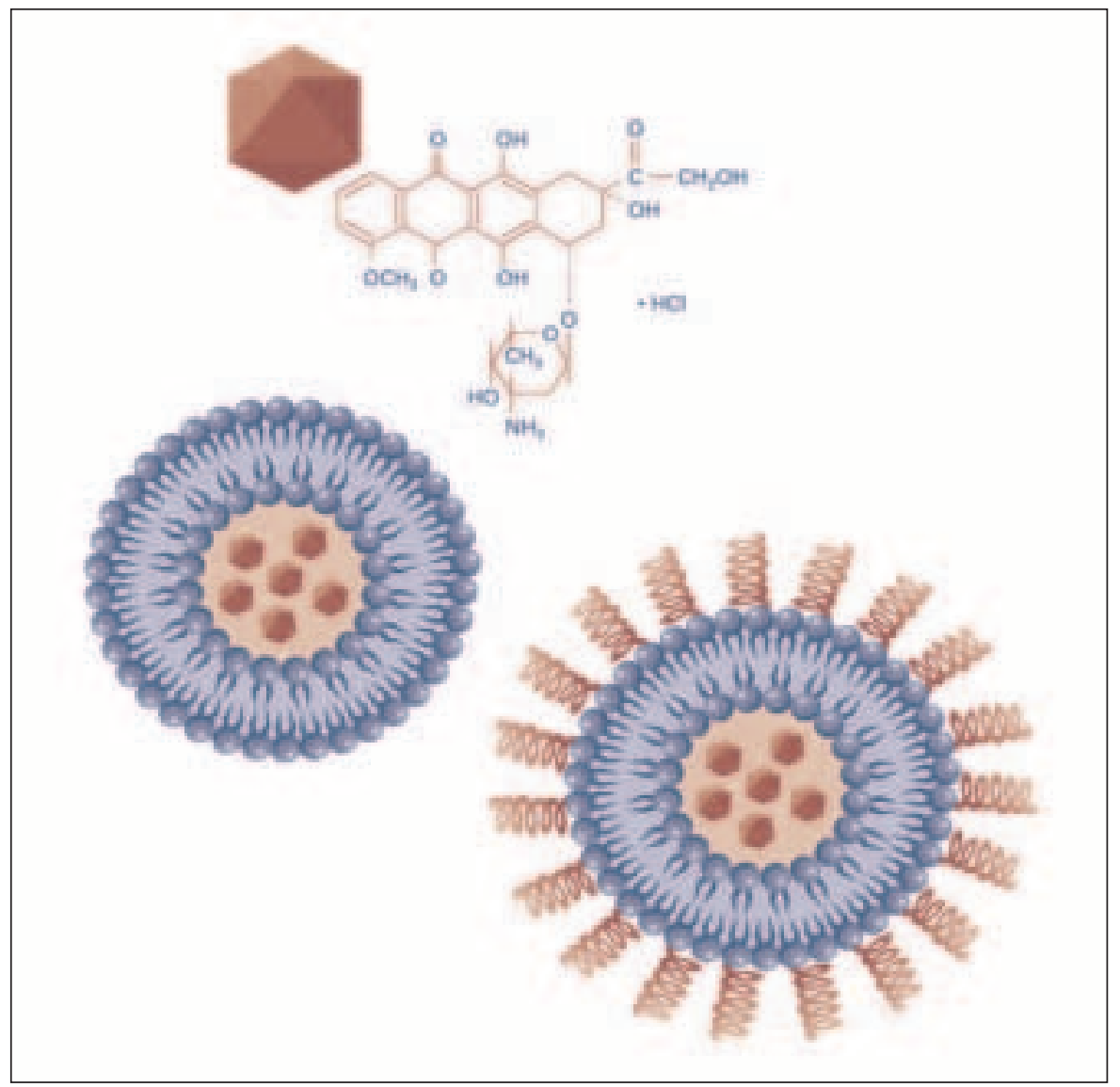

Abb. 1. Caelyx: pegyliertes liposomales Doxorubicin. die kardialen Nebenwirkungen einer Anthrazyklin-Therapie noch Monate bis Jahre nach der Behandlung auftreten können. Vor diesem Hintergrund ist der Einsatz des pegylierten liposomalen Doxorubicins von erheblicher Bedeutung. In Studien konnte gezeigt werden, dass die Pegylierung des Liposoms die Verweildauer des Doxorubicins im Plasma so steigert, dass das Nebenwirkungsprofil völlig verändert ist [Gabizon et al., 1994; Safra et al., 2001] Kardiotoxizitäten werden in Folge kaum noch beobachtet. «Selbst bei einer Applikation von hohen Anthrazyklindosen bis zu $1000 \mathrm{mg} / \mathrm{m}^{2}$ ist die gefürchtete Herzwirkung nahezu komplett vermeidbar», fügte Bokemeyer an. Darüber hinaus weist die liposomale Form ein deutlich reduziertes Risiko für Nekrosen bei Paravasationen auf. Ein nicht zu unterschätzender Vorteil für die Lebensqualität der Patientinnen ist zudem, dass es unter Caelyx äußerst selten zu Haarausfall, zu Myelosuppression oder zu Übelkeit und Erbrechen kommt. «Insgesamt stellt Caelyx eine klare Bereicherung für die onkologische Therapie dar», betonte der Tübinger Onkologe.

\section{Gute Wirksamkeit beim Mamma- karzinom}

Caelyx ist bereits seit einigen Jahren zur Behandlung beim AIDS-assoziierten Kaposi-Sarkom und beim fortgeschrittenen Ovarialkarzinom zugelassen. Die Ergebnisse einer Vergleichsstudie mit der pegylierten liposomalen Formulierung und konventionellem Doxorubicin bei Patientinnen mit metastasiertem Mammakarzinom haben nun zur Zulassung auch in dieser Indikation geführt. Privatdozent Dr. Gunter von Minckwitz vom Universitätsklinikum Frankfurt am Main erläuterte, dass die Daten und Resultate dieser Phase III-Studie deutlich die Vorteile von Caelyx wie die geringere Kardiotoxizität bei Äquieffektivität belegen [Wigler et al., 2002].

In der randomisierten Studie bei insgesamt 509 Frauen mit metastasiertem Brustkrebs erhielten 255 Patientinnen konventionelles Doxorubicin in einer Dosierung von $60 \mathrm{mg} / \mathrm{m}^{2}$ Körperoberfläche alle drei Wochen. 254 Patientinnen bekamen alle vier Wochen $50 \mathrm{mg} / \mathrm{m}^{2}$ des pegylierten liposomalen Doxorubicins. Das dadurch erzielte progressionsfreie Überleben unterschied sich unter Caelyx mit 6,9 versus 7,8 Monaten nicht signifikant. Auch die durchschnittliche Gesamtüberlebenszeit war mit 22 versus 21 Monaten vergleichbar. Signifikante Unterschiede gab es hingegen bei der Kardiotoxizität: Bei 48 der mit konventionellem Doxorubicin behandelten Frauen, aber nur bei 10 derjenigen Frauen, die pegyliertes liposomales Doxorubicin erhielten, nahm die linksventrikuläre Ejektionsfraktion deutlich ab $(\mathrm{p}<0,001)$. Subgruppenanalysen zeigen, dass ältere Patientinnen einem doppelt so hohem Risiko für das Auftreten einer Kardiotoxizität unter konventionellem Doxorubicin ausgesetzt sind. «Noch deutlicher zeigt sich der Vorteil durch das pegylierte liposomale Doxorubicin, wenn Patientinnen mit einer Anthrazyklin-Vorbehandlung betrachtet werden», so von Minck- 
Tab. 1. Kardiotoxizität (Wigler et al., 2002)

\begin{tabular}{lllll}
\hline & $\mathrm{n}$ & Kardiale Ereignisse & HR & 95\% CI für HR \\
\hline $\begin{array}{l}\text { Alter } \geq \text { 55 Jahre } \\
\quad \text { Caelyx }\end{array}$ & 159 & 6 & 2,04 & $0,81-5,18$ \\
$\quad$ Doxorubicin & 152 & 18 & & \\
$\quad \begin{array}{l}\text { Vorbehandlung mit Anthrazyklinen } \\
\quad \text { Caelyx }\end{array}$ & 38 & 1 & 7,27 & $0,93-56,80$ \\
$\quad$ Doxorubicin & 40 & 11 & & \\
$\quad$ Kardialer Risikofaktor & 122 & 5 & 2,7 & $1,01-7,18$ \\
$\quad$ Caelyx & 121 & 21 & & \\
$\quad$ Doxorubicin & & & & \\
\hline
\end{tabular}

Tab. 2. Progessionsfreies Überleben (Keller et al., 2001)

\begin{tabular}{|c|c|c|c|c|c|}
\hline & \multirow[t]{2}{*}{$\mathrm{n}$} & \multicolumn{2}{|c|}{$\begin{array}{l}\text { Medianes progessionsfreies } \\
\text { Überleben, Monate }\end{array}$} & \multirow[t]{2}{*}{ HR } & \multirow[t]{2}{*}{$95 \% \mathrm{CI}$} \\
\hline & & Caelyx & Vergleichsarm & & \\
\hline \multicolumn{6}{|c|}{ Anthrazyklin-vorbehandelt } \\
\hline Ja & 250 & 2,43 & 2,66 & 1,09 & $0,83-1,43$ \\
\hline Nein & 51 & 4,96 & 1,91 & 2,05 & $1,30-4,82$ \\
\hline \multicolumn{6}{|c|}{ Anthrazyklin-resistent } \\
\hline $\mathrm{Ja}$ & 112 & 2,56 & 2,37 & 1,20 & $0,80-1,82$ \\
\hline Nein & 186 & 3,29 & 2,33 & 1,31 & $0,95-1,81$ \\
\hline
\end{tabular}

Tab. 3. Zytostatische Therapie nach Anthrazyklinen und Taxanen

\begin{tabular}{ll}
\hline Empfehlungsgrad: B & Evidenzniveau* \\
\hline Experimentelle Therapien in & \\
Studien & I \\
Capecitabine & III \\
CMF & III \\
Gemcitabine & III \\
Pegyliertes, liposomales & \\
$\quad$ Doxorubicin & I \\
Taxane & III \\
Vinorelbine & I \\
\hline
\end{tabular}

*Alphabetisch und nicht nach Effektivität sortiert.

www.ago-online.de.

witz weiter. «Bei diesem Patientinnenkollektiv ist das Risiko einer Kardiotoxizität unter konventionellem Doxorubicin um den Faktor 7,3 erhöht.»

Die guten Studienergebnisse haben - so der Referent abschließend - inzwischen auch dazu geführt, dass das pegylierte liposomale Doxorubicin für die Therapie nach Anthrazyklinen und Taxanen in die Standardempfehlungen der Organkommission Mammakarzinom der Arbeitsgemeinschaft Gynäkologische Onkologie (AGO) der Deutschen Krebsgesellschaft aufgenommen wurde (Tab. 3).

\section{Geeigneter Kombinationspartner bei neuen Therapieansätzen}

Nach Auffassung von PD Dr. Hans-Joachim Lück, Universitätsklinikum Hannover, gewinnt Caelyx zusätzlich an Bedeutung, da eine zunehmende Zahl an Patientinnen bereits mit Anthrazyklinen vorbehandelt sind. Diesen kann ohne nennenswerte Steigerung der Kardiotoxizität dann das pegylierte liposomale Doxorubicin verabreicht werden. Immerhin wurden Ansprechraten von bis zu $31 \%$ bei diesen stark vorbehandelten Patientinnen erzielt [Ranson et al., 1997]. Aber nicht nur in der Monotherapie, sondern auch in der Kombinationstherapie, scheint Caelyx laut bisherigen Studiendaten gut einsetzbar zu sein. Das pegylierte liposomale Doxorubicin wird als Kombinationspartner für mehrere andere Substanzen wie Trastuzumab, Docetaxel, Gemcitabine oder auch Vinorelbin in klinischen Studien überprüft. Solche Therapieansätze können zukünftig immer dann eine Rolle spielen, wenn ein hoher Remissionsdruck Kombinationstherapien verlangt.

\section{Caelyx hat Spektrum beim Mamma- karzinom deutlich erweitert}

Professor Dr. Manfred Kaufmann vom Universitätsklinikum Frankfurt/Main verwies darauf, dass die pegylierte liposomale Formulierung auch bei Patientinnen, die auf eine Taxan-haltige Therapie nicht angesprochen hatten, eine zufriedenstellende Effektivität zeigt [Keller et al., 2001]. Zudem deuten Daten darauf hin, dass eine Kreuzresistenz von Caelyx bei Anthrazyklin-resistenten Patientinnen fehlt. Der Einsatz der Substanz in der First-line-Therapie oder auch in der kurativen Situation wird laut Kaufmann künftig zu klären sein. «Zum momentanen Zeitpunkt steht jedoch schon fest, dass die neue und trotzdem bereits bewährte Substanz das Spektrum in der Therapie des Mammakarzinoms deutlich erweitert hat», stellte der Frankfurter Krebsspezialist fest. Von einer Therapie mit Caelyx profitieren, so Kaufmann, vor allem ältere Patientinnen, Patientinnen mit bereits bestehenden kardialen Risikofaktoren oder einer präexistenten Herzerkrankung sowie Patientinnen mit einer vorausgegangenen Anthrazyklin-Behandlung oder einer mediastinalen Bestrahlung. Abschließend fasste Kaufmann zusammen: "Caelyx ist sicherlich keine Wunderwaffe. Aber es handelt sich um eine wundervolle Erfindung und exzellente Substanz, die getarnt als «Trojanisches Pferd» Effektivität, aber auch gute Verträglichkeit beim Mammakarzinom verspricht.»

\section{Literatur}

Gabizon A et al.: Cancer Research 1994;54;987-992. Keller A N et al.: Proc Am Soc Clin Oncol 2001;20:abstr. 115. Ranson MR et al.: J Clin Oncol 1997;15:3185-3189. Safra T et al.: Cancer 2001:91:90-100.

Wigler N et al.: Proc Am Soc Clin Oncol 2002;21:abstr. 177

\section{Impressum}

Caelyx beim metastasierten Mammakarzinom: Neue effektive Therapieoption mit signifikant verringerter Kardiotoxizität

Pharma Forum in Onkologie 26/2/03

Deutschland

S. Karger $\mathrm{GmbH}$

Lörracher Straße 16a

D-79115 Freiburg

Tel. +49761452070

Fax +497614520714

E-mail Information@Karger.de

Übrige Länder

S. Karger AG

Allschwilerstrasse 10

CH-4009 Basel

Tel. +4161306 1111

Fax +41613061234

E-mail Karger@Karger.ch

Mit freundlicher Unterstützung der Essex Pharma $\mathrm{GmbH}$.

Der Verlag und die Herausgeber der Zeitschrift übernehmen keine Verantwortung für diese Rubrik.

(C) 2003 by S. Karger Verlag

Verlag für Medizin und Naturwissenschaften $\mathrm{GmbH}$ Lörracher Straße 16a, D-79115 Freiburg

Die Wiedergabe von Gebrauchsnamen, Handelsnamen, Warenbezeichnungen usw. in dieser Zeitschrift berechtigt auch ohne besondere Kennzeichnung nicht zur Annahme, dass solche Namen im Sinne der Warenzeichen- und Markenschutz-Gesetzgebung als frei zu betrachten wären und daher von jedermann benutzt werden dürfen. Für Angaben von Dosierungsanweisungen und Applikationsformen kann vom Verlag keine Haftung übernommen werden. Derartige Angaben müssen vom jeweiligen Anwender im Einzelfall anhand anderer Literaturstellen auf ihre Richtigkeit überprüft werden 